Research Article

\title{
Integrating TTC-SC5 and TTC-SC6 “Shared-Clock” Protocols
}

\author{
Muhammad Amir ${ }^{1 *}$, Syed Waqar Shah ${ }^{1}$, Salman Ilahi ${ }^{1}$ and Michael J. Pont ${ }^{2}$ \\ ${ }^{1}$ Department of Electrical Engineering, UET, Peshawar, Khyber Pakbtunkhwa, Pakistan; ${ }^{2}$ SafettySystems ${ }^{T M}$ Ltd, Registered \\ Office, 15 Nether End, Great Dalby, LE14 2EY, UK.
}

Abstract: In recent past, to rectify certain limitations of bus-based "Shared-Clock" (SC) architectures we have
developed two new SC protocols based on star topology. In both bus and star-based designs, the Controller
Area Network (CAN) protocol was used for network communications. Previously we have demonstrated
that both new protocols in their individual capacities have the potential for addressing issues relating to
Time-Triggered Cooperative (TTC) scheduling, Time Triggered bus-based CAN networks and the Single
Point of Failure (SPF) hypothesis in star networks. In this paper, we present a mechanism for integrating the
properties of both protocols in one. We present an amalgamation of protocols that result in a new protocol
which addresses all the above stated issues on a single platform.
Received: January 10, 2020; Accepted: May 20,2020; Published:June 20,2020
"Correspondence: Muhammad Amir, Department of Electrical Engineering, UET, Peshawar, Khyber Pakhtunkhwa, Pakistan; Email: amir@
nwfpuet.edu.pk
Citation: Amir, M., S.W. Shah, S. Ilahi and M.J. Pont. 2020. Integrating TTC-SC5 and TTC-SC6 "Shared-Clock" protocols. Journal of
Engineering and Applied Sciences, 39(1):35-40.
DOI: http://dx.doi.org/10.1758/journal.jeas/39.1.35.40
Keywords: Shared-Clock protocols, Controller Area Network (CAN), Differential tick rate, Fault-management, Industrial automation

\section{Introduction}

I n our past research, we have considered various ways for reliably employing TT architectures using low-cost embedded components (Pont, 2001, 2003; Pont and Banner, 2004; Ayavoo et al., 2005; Amir and Pont, 2010; Amir et al., 2010). Both single and multiprocessor designs were a part of this work. In the case of multi-processor designs, we have sought to demonstrate that "Shared-Clock" (SC) architecture provides a simple, flexible platform for many systems (Pont, 2001). In such designs, the Controller Area Network (CAN) protocol introduced by Robert Bosch $\mathrm{GmbH}$ in the 1980s (Bosch, 1991) provides high reliability communications at low cost (Farsi and Barbosa, 2000; Fredriksson, 1994; Thomesse, 1998; Sevillano et al., 1998). Since the CAN protocol has become widely used in many sectors, such as automotive and automation (Farsi and Barbosa,
2000; Fredriksson, 1994; Thomesse, 1998; Sevillano et al., 1998; Pazul, 1999; Zuberi and Shin, 1995; Misbahuddin and Al-Holou, 2003), most modern microprocessor families now have members with single and multiple on-chip support for this protocol e.g., (Philips, 1996, 2004; Siemens, 1997; Infineon, 2004; NXP, 2020).

The SC protocols work on a Master-Slave(s) arrangement. Originally we introduced two SC protocols in 2001 (Pont, 2001). They are best known as ("TTC-SC1" and "TTC-SC2"), where TTC-SC means "Time Triggered Cooperative Shared Clock". In later papers we introduced four new protocols ("TTC-SC3", “TTC-SC4", "TTC-SC5" and "TTCSC6") which were a better match for the needs of some applications (Ayavoo et al., 2005; Amir and Pont, 2010; Amir et al., 2010). All implementations from TTC-SC1 till TTC-SC4 were based on CAN 
bus topology. We have also introduced a "Dual CAN" bus topology based SC design for utilization in highreliability applications (Short and Pont, 2007). TTCSC5 and TTC-SC6 on the other hand were deployed using a CAN based star topology in order to enhance the capabilities of SC architectures. The star topologies which were designed for such implementations were produced using the multi-CAN support offered on the Master node microcontrollers (NXP, 2020). Star-based topologies are felt to offer a number of advantages for high-reliability embedded systems e.g., (Manuel et al., 2006; FlexRay, 2004; TTA, 2003; Manuel et al., 2005).

In the case of time-triggered designs, they also offer the possibility that we can use the star configuration to create a very flexible design in which periodic tasks operate at a range of independent "Tick rates" (with a different rate supported on each arm of the star), as shown in Figure 1 and (Amir and Pont, 2010). This type of configuration has the potential to address some of the limitations of single-processor TTC designs, and is difficult to achieve in a design based on a single bus. Star topologies in SC environments also have the capability of satisfying the CAN faultmodel through an arrangement as shown in Figure 2 and (Amir and Pont, 2010).

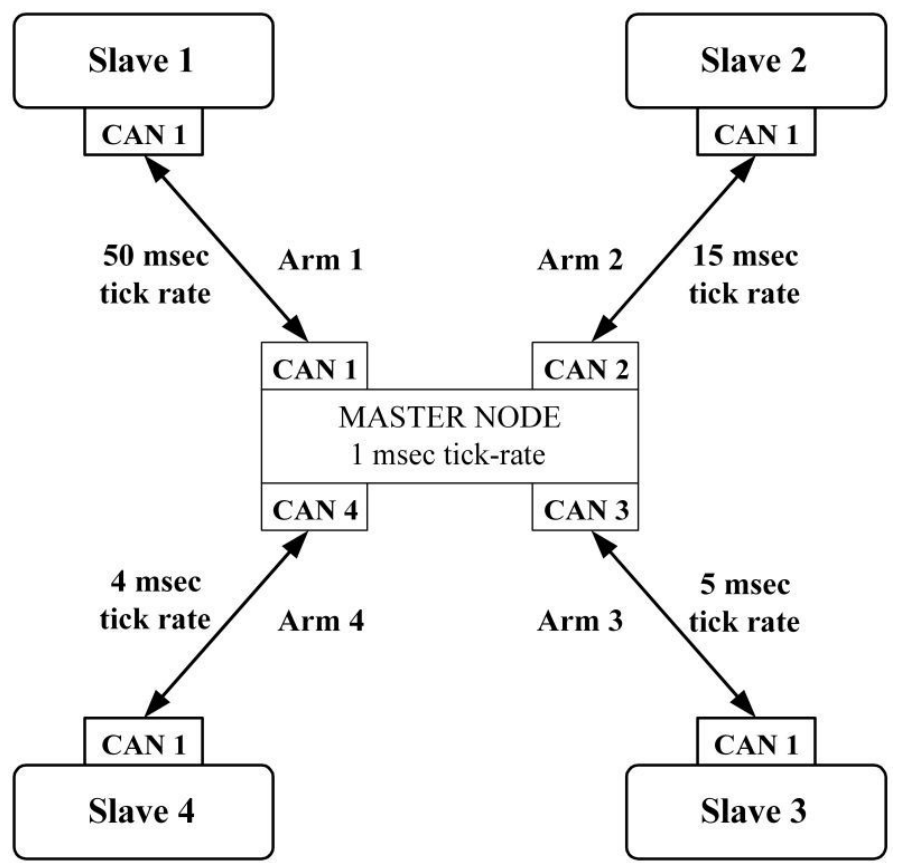

Figure 1: TTC-SC5 CAN-based star topology (Differentialtick rate).

In this paper, we present a possible combination of the individual properties of TTC-SC5 and TTCSC6 in a single hybrid protocol known as TTC-SC7.
The paper is organised as follows: In the following section, we present the internal strategy of our TTCSC5 protocol. The section after that highlights the techniques used in TTC-SC6 architecture. In the second last section, we propose the amalgamation of TTC-SC5 and TTC-SC6 into TTC-SC7and finally, we present our conclusions.

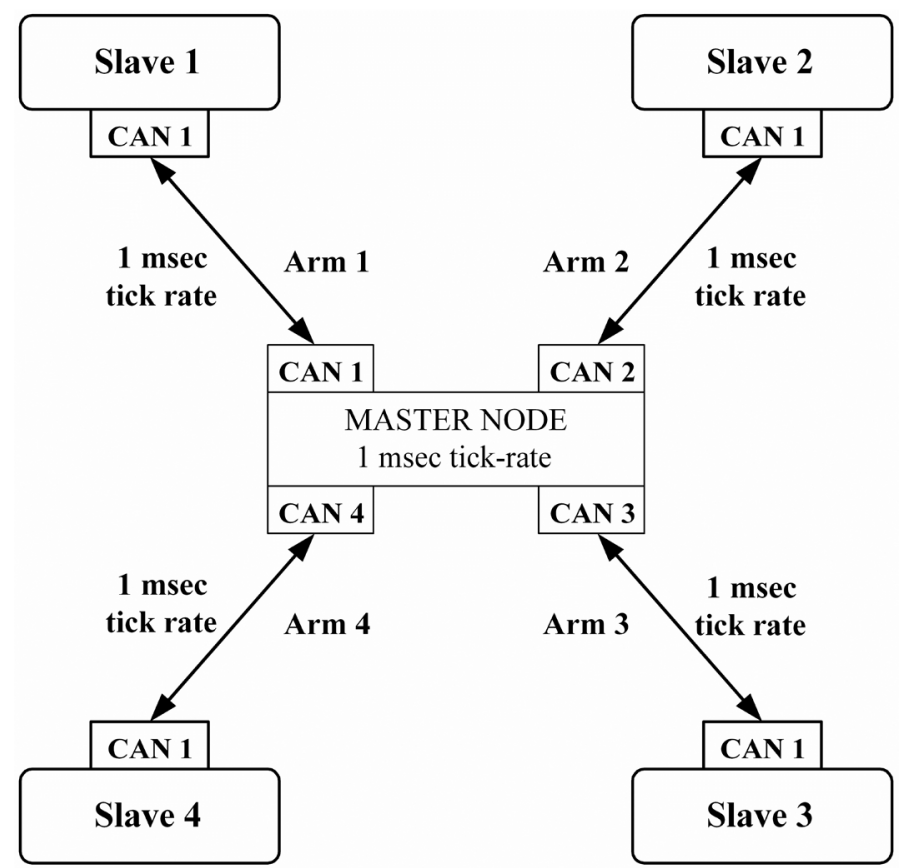

Figure 2: TTC-SC6 (Single Tick rate) for CAN fault-model.

\section{TTC-SC5 protocol}

In this section, we highlight all the features of TTCSC5 algorithm and give a description of ways in which this protocol achieved them.

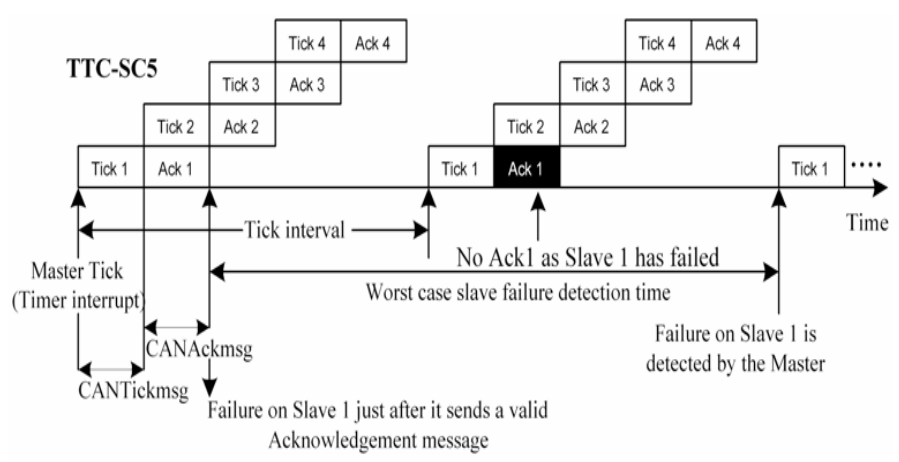

Figure 3: TTC-SC5 single rate Tick transmission strategy.

\section{Tick transmission (Single rate) strategy}

In TTC-SC5, the Master node (NXP, 2020) has got four separate CAN interfaces connecting four Slave nodes through CAN cables. The Master node here is configured to send a Tick message and receive an Ack message on each of the four CAN links. This means that if the overall Tick interval (duration between two successive timer interrupts on the Master node) of 
the network is kept at $1 \mathrm{msec}$, during that $1 \mathrm{msecthe}$ Master node have to send each Slave an individual Tick message and receive an Ack message back from each Slave (see Figure 3). The overall number of messages in $1 \mathrm{msec}$. in this case is eight. During the next Tick interval, the Master node checks the Ack messages received earlier before sending out four new Tick messages. This cycle keeps repeating itself after initialization. Due to the use of a Star topology no TDMA sequencing as in bus-based protocols is required. In this algorithm both the Tick and Ack messages carry data. The reception of Ack messages are also used for error detection in Slaves. In previous bus-based SC designs to make the Tick and Ack messages identifiable on the bus, a particular Slave ID was to be inserted in them. In TTC-SC5 as the Master node is connected to the Slaves in a Star topology it does not have to cycle through the Slave IDs. In this case the Tick messages generate interrupts on the Slaves thus causing the Slave nodes to generate Ack messages with or without a Slave ID inside the Tick message in the first place. So in TTC-SC5 environment, an insertion of a Slave ID in the Tick message is not essential and this makes the protocol less complex. The Tick and Ack messages can be naturally identified by the Master and Slaves due to the use of separate CAN links of the Star topology. The Tick transmission in TTC-SC5 can be best understood from Listing 1.

\section{Tick transmission (Differential rate) strategy}

TTC-SC5 also offers the possibility that we can use the star configuration to create a very flexible design in which periodic tasks operate at a range of independent "Tick rates" (with a different rate supported on each arm of the star). This type of configuration has the potential to address some of the limitations of singleprocessor TTC designs, and is difficult to achieve on a bus-based design. TTC-SC5 achieves this behaviour by making use of the Star-based topology. In previous bus-based designs the Master node used to send Tick messages from its scheduler's "Update function". In TTC-SC5, the Tick message transmission functions can be added as tasks to the Master scheduler as shown in Listing 2.

In Listing 2, the initial delay for all the added tasks is kept at 0 . While the TICK PERIOD on each of the four arms of the star can be set according to the scheduling needs of the task-set running on four Slave nodes. Remember that each Slave is connected to the Master via an independent CAN link. In this way Tick message transmission can be scheduled to support a different Tick rate on each arm of the star network. In this approach the Master scheduler runs at a fundamental Tick rate $(1 \mathrm{msec}$.) and sends Tick messages on each arm of the star with rates that are multiples of the fundamental Tick rate. Please note that each added task in Listing 2 corresponds to an individual CAN interface on the Master node.
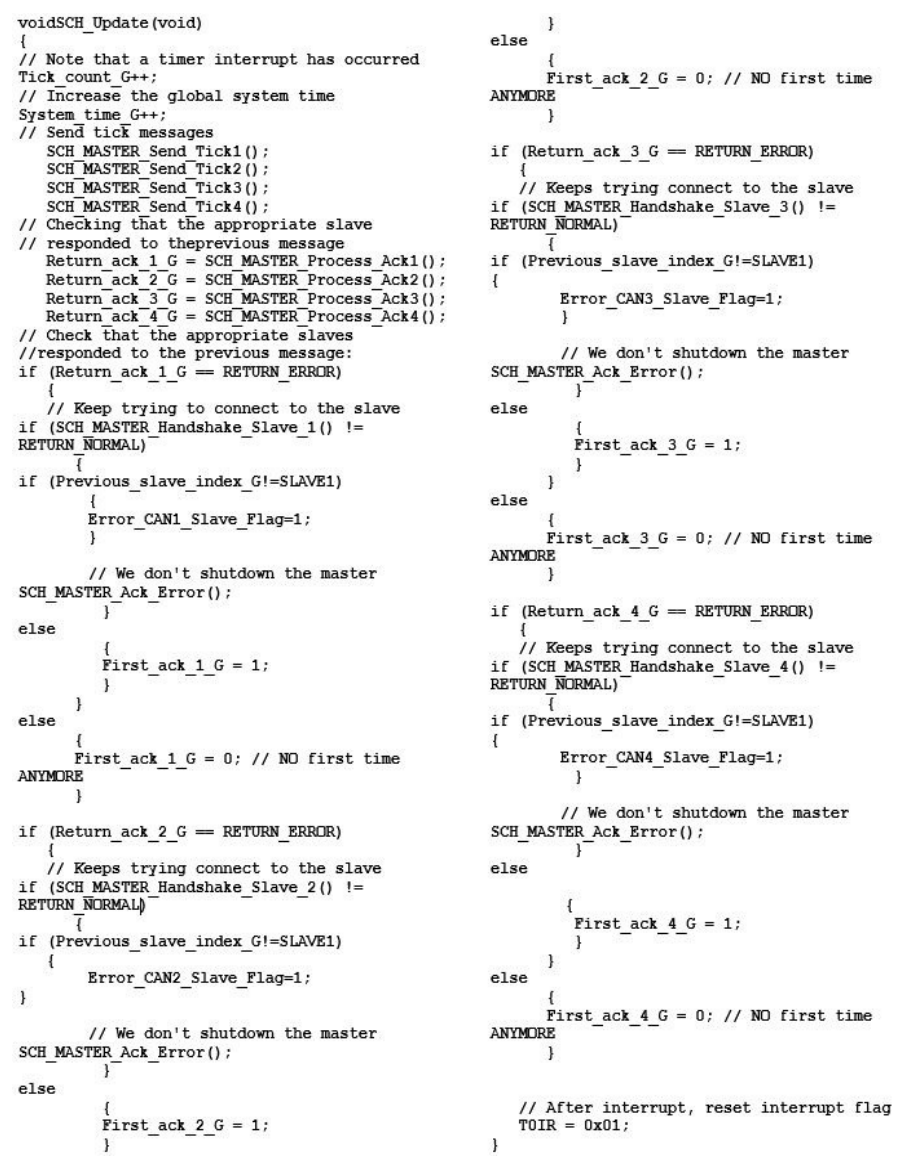

Listing 1: Routine for single Tick rate in TTC-SC5.

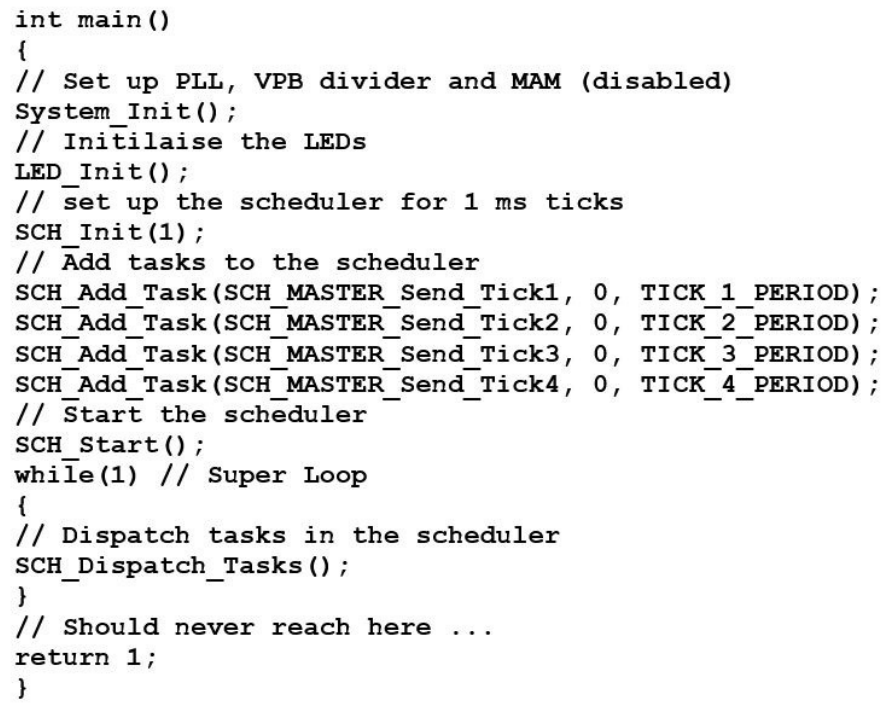

Listing 2: Routine for different Tick rates on individual arms of the star topology. 


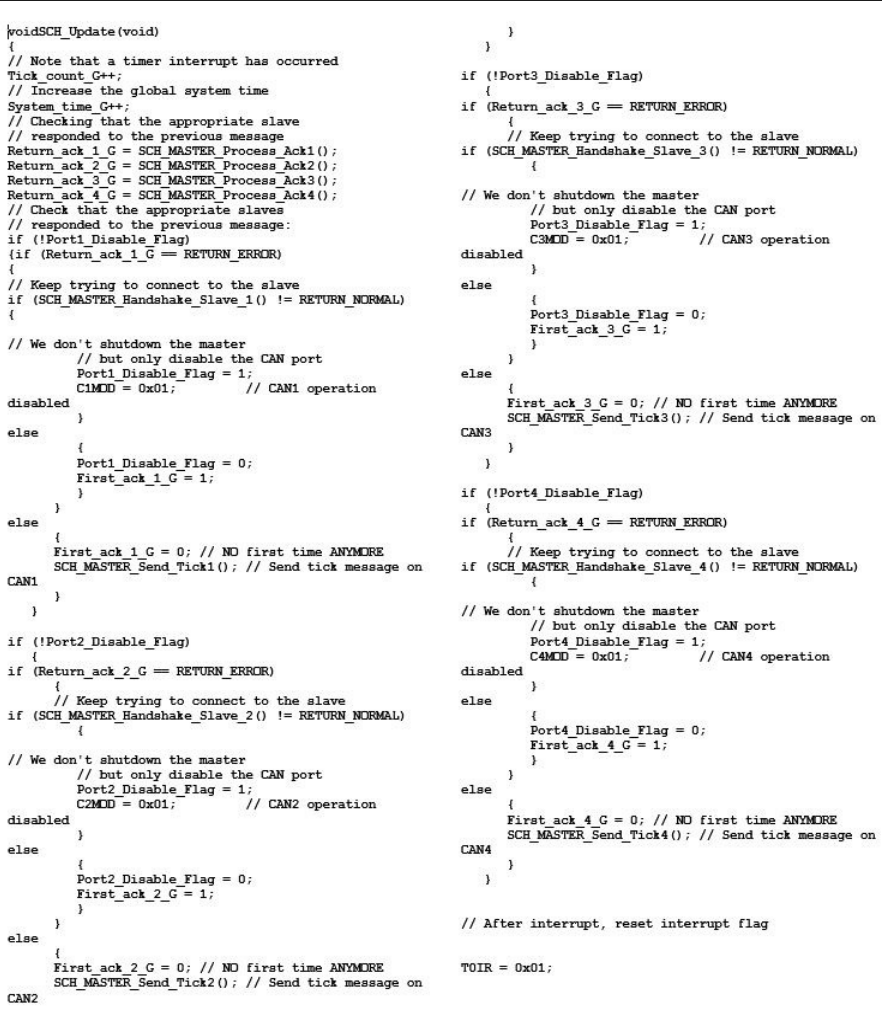

Listing 3: Routine for fault-confinement in TTC-SC6.

\section{TTC-SC6 protocol}

In this section, we highlight all the features of TTCSC5 protocol and give a description of ways in which this protocol achieved them.

\section{Tick transmission strategy}

TTC-SC6 can incorporate both single rate and differential rate Tick transmission strategies but the initial implementation uses single Tick rate. In TTCSC6 differential tick rate mechanism is achieved with no scheduler overhead increase from TTC-SC5.

\section{Addressing the CAN and TTC-SC6 fault-model}

The related fault-model with TTC-SC6 architecture and its confinement are given in Table 1. The provisions in TTC-SC6 for tackling such a faultmodel are inserted directly into the "Scheduler Update" function as shown in Listing 3. This shows that without assigning additional hardware or software resources, fault confinement can be achieved cost-effectively from within the protocol itself. The main reason for such fault confinement is the fact that this $\mathrm{SC}$ protocol is deployed using a CAN-based star topology (Listing 3).

\section{Proposed TTC-SC7 hybrid protocol}

We present an amalgamation of TTC-SC5 and TTC-SC6protocols in this section. Here, we show a possibility of having a single protocol as a result.

\section{Tick transmission strategies}

The Tick transmission strategy used in any SC protocol depends upon the application that it caters for. The proposed TTC-SC7 protocol can be used with the option of both single and differential Tick rates. As both the strategies have been used previously in TTC-SC5, so there is no reason why they cannot be used in the same manner inside the TTC-SC7 hybrid protocol.

Table 1: Fault-model for CAN and associated TTC-SC6 protocol.

\section{Fault-Model (CAN and TTC-SC6)}

\section{Origin Fault Description}

CAN hard- Node stuck at fault (Master/ Stuck at dominant bit

ware Slave) Stuck at recessive bit

Network partitioning fault (Single or multiple link severing) Shorted medium fault (Single or multiple links)

Network Master hardware reset (accidental or due to power fluctuanode tion)

Slave(s) hardware reset (accidental or due to power fluctuation)

Data corruption due to Master or Slaves hardware

Scheduler Software problem on the Master or Slave nodes in sending Tick or Acknowledgement messages

Data corruption on CAN links of the star topology due to external or internal factors. Data corruption will prevent the schedulers from interpreting the data correctly.

Single point Master node failure (Single point of failure hypothesis) of failure

\section{Confinement in TTC-SC6}

Port disablement (Silent or babbling removal)

Port disablement (Silent or babbling removal)

Port(s) disablement (not effecting other nodes)

Port(s) disablement (not effecting other nodes)

The Slaves go into safe state and wait for Master to resume communication

The Master node tries to reconnect to the effected Slave(s). If unsuccessful then that Slave(s) is isolated from the rest of the network (Port(s) disablement)

Port(s) disablement (not effecting other nodes)

Port(s) disablement (not effecting other nodes)

The Master node tries to reconnect to the effected Slave(s). If unsuccessful then that Slave(s) is isolated from the rest of the network (Port(s) disablement)

Backup Master takes over the network 
Addressing the overall $S C$ fault-model

The fault confinement mechanism in TTC-SC6 for tackling CAN and SC protocol based faults was made a part of the scheduler. The mechanism was made effective due the fact that the protocol was deployed using a CAN based star topology. TTC$\mathrm{SC} 7$ is also proposed for the same architecture, so the provisions for fault-confinement can be easily inserted on a scheduler level. In addition to that, the TTC-SC7 protocol is been also equipped with fault-tolerance capabilities as shown in Figure 4 through pseudo code. SWT in Figure 4 stands for "System's Wait Time" which is used for checking whether a fault is intermittent or permanent. In case of an intermittent fault, TTC-SC7 waits to see if the fault will go away (Note: duration of SWT is in the hands of the system's designer). If the wait exceeds the SWT then TTC-SC7 engages a nonfaulty peripheral node through its redundancy management algorithm.

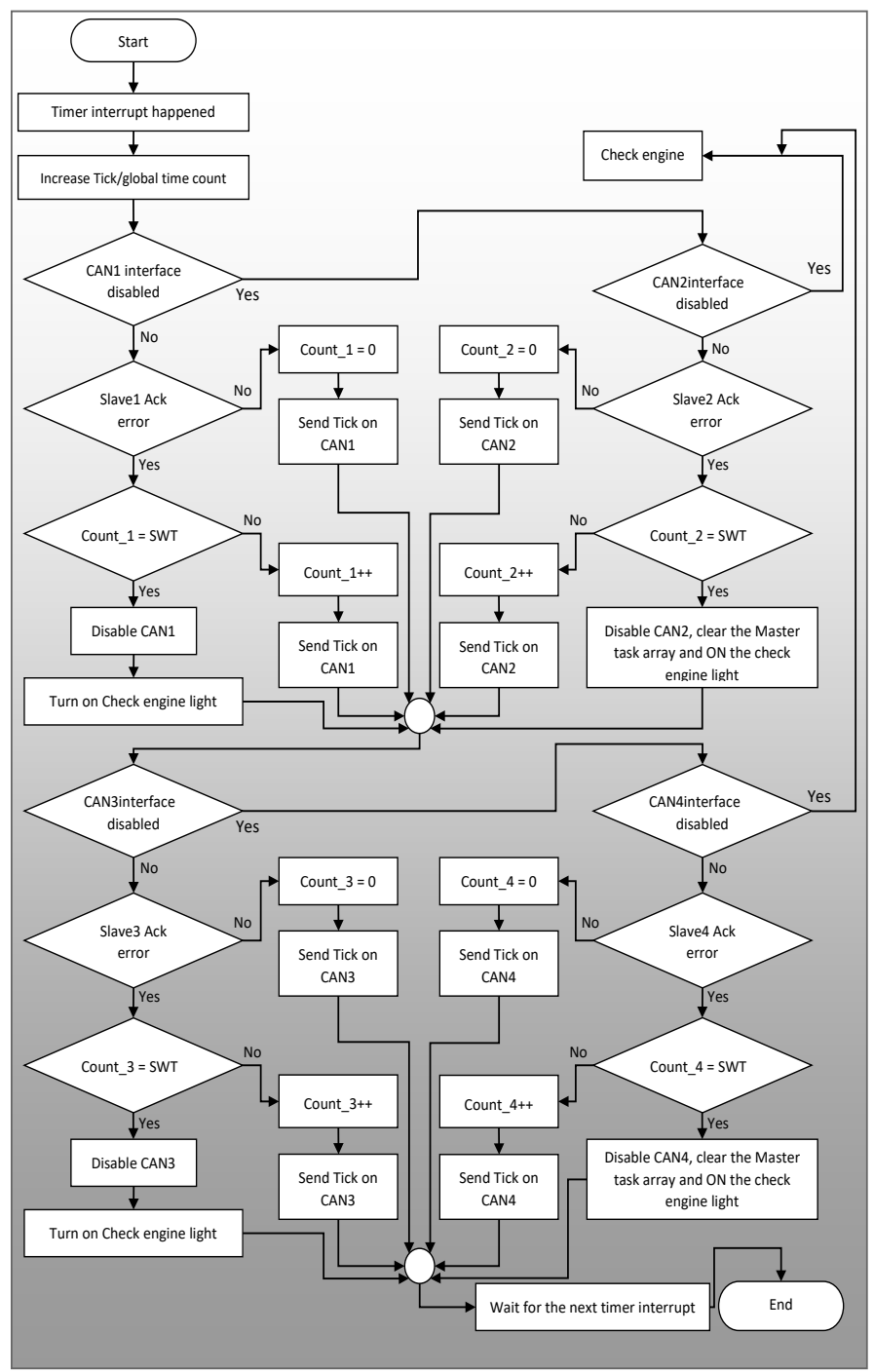

Figure 4: Pseudocode for the Port Guardian mechanism in TTCSC7 protocol.
It is pivotal to note here that all characteristics of TTC-SC5 and TTC-SC6 are interchangeable as shown in Figure 5 and such characteristics can be amalgamated in order to create a protocol such as TTC-SC7 with all individual properties.

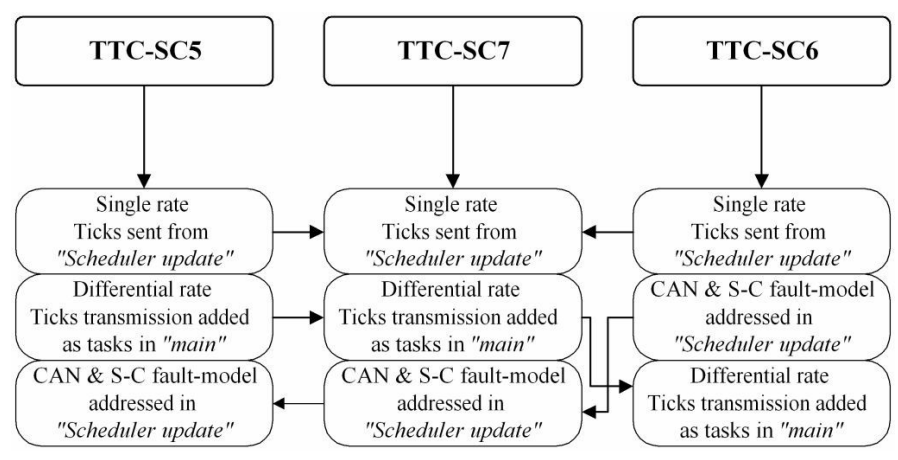

Figure 5: Creating TTC-SC7 by amalgamating TTC-SC5 and $T T C-S C 6$.

\section{Conclusions and Recommendations}

Both TTC-SC5 and TTC-SC6 evolved due to the experience gained with the previous four bus-based $\mathrm{SC}$ algorithms through implementing them through the RapidiTTy software plat form (RapidiTTy, 2020). In their own capacity they can be deployed for specific applications like enhancing flexibly and faultconfinement.

But if certain applications in embedded systems require the properties of TTC-SC5 and TTC-SC6 in a combine manner then both mentioned protocols can be completely integrated with considerable ease and simplicity. The result of such an amalgamation is the proposed TTC-SC7 protocol shown in Figure 5.

\section{Novelty Statement}

The proposed protocol i.e. TTC-SC7 provides flexibility and fault-management in Time-Triggered applications through a single platform.

\section{Author's Contribution}

The design of TTC-SC5 \& TTC-SC6 Shared-Clock protocols was carried out by Muhammad Amir. The idea of integrating these two protocols came from Syed Waqar Shah. The verification of software efficiency/optimization was done by Salman Ilahi and finally, the feasibility of the overall proposed design was validated by Michael J. Pont 
Conflict of interest

The authors have declared no conflict of interest.

\section{References}

Amir, M. and M.J. Pont. 2010. A time-triggered communication protocol for CAN-based networks with a fault-tolerant star topology. Proceedings of international symposium on advanced topics on embedded systems and applications (ESA2010), in conjunction with IEEE international conference on embedded software and systems, Bradford, UK.

Amir, M., D. Ayavoo and M.J. Pont. 2010. A novel shared-clock protocol for fault-confinement in can-based distributed systems. Proceedings of the $5^{\text {th }}$ IEEE system of systems conference, Loughborough, UK.

Ayavoo, D., M.J. Pont, M.J. Short and S. Parker. 2005. Two novel shared-clock scheduling algorithms for use with CAN-based distributed systems. Proceedings of the $2^{\text {nd }}$ UK Embedded Forum, Birmingham, UK, pp. 246-261.

Bosch, R.G., 1991. CAN specification version 2.0. Robert Bosch GmbH, Postfach 50, D-7000 Stuttgart 1, Germany.

Farsi, M. and M. Barbosa. 2000. CAN open implementations: Applications to industrial networks. Exeter: Research Studies Press Ltd.

Flex-Ray, 2004. FlexRay communication system protocol specification version 2.0. FlexRay Consortium.

Fredriksson, L.B., 1994. Controller area networks and the protocol CAN for machine control systems. Mechatronics, 4(2): 159-192. https:// doi.org/10.1016/0957-4158(94)90041-8

NXP, 2020. http://www.nxp.comaccessed on 14/11/2020 at 23:00 PST.

https://rapiditty-lite.software.informer.com/ download/ accessed on 14/11/2020 at 23: 00 PST.

Infineon, 2004. Connecting C166 and C500 microcontroller to CAN.Infineon Technologies.

Manuel, B., P. Julián, N. Guillermo and A. Luís. 2006. An active star topology for improving fault confinement in CAN networks. IEEE Trans. Industr. Inform., 2(2): 78-85. https:// doi.org/10.1109/TII.2006.875505

Manuel, B., A. Luís and P. Julián. 2005. ReCAN centrate: A replicated star topology for CAN networks. 0-7803-9402-X/05/\$20.00@2005 IEEE, Vol. 2.

Misbahuddin, S. and N. Al-Holou. 2003. Efficient data communication techniques for Controller Area Network (CAN) protocol. ACS/IEEE Int. Conf. Comput. Syst. Appl., Tunis, Tunisia.

Pazul, K. 1999. Controller Area Network (CAN) basics. Microchip Technology Inc.

Philips, 1996. PCA82C250/251 CAN transceiver. Philips semiconductors.

Philips, 2004. SJA1000 stand-alone CAN controller.

Pont, M.J., 2001. Patterns for time-triggered embedded systems: Building reliable applications with the 8051 family of microcontrollers. Harlow: Addison Wesley/ ACM Press.

Pont, M.J., 2003. Supporting the development of time-triggered co-operatively scheduled (TTCS) embedded software using design patterns. Informatica, 27(1): 81-88.

Pont, M.J. and M.P. Banner. 2004. Designing embedded systems using patterns: A case study. J. Syst. Softw., 71(3): 201-213. https://doi. org/10.1016/S0164-1212(03)00006-2

Sevillano, J.L., A. Pascual, G. Jimenez and B. Civit. 1998. Analysis of channel utilization for Controller Area Networks. Comput. Commun., 21(16): 1446-1451. https://doi.org/10.1016/ S0140-3664(98)00166-2

Short, M.J. and M.J. Pont. 2007. Fault-tolerant time-triggered communication using CAN. IEEE Trans. Ind. Inf., 3(2): 131-142. https:// doi.org/10.1109/TII.2007.898477

Siemens, 1997. Proceedings of the European pattern languages of programming conference.

Thomesse, J.P., 1998. A review of the field buses. Ann. Rev. Contr., pp. 35-45. https://doi. org/10.1016/S1367-5788(98)00003-0

TTA-Group, 2003. Time-triggered protocol TTP/C High-Level Specification Doc. Protocol Ver. 1.1, 1.4.3 ed. Vienna, Austria, TTTECH.

Zuberi,K.M. and K.G.Shin.1995.Non-preemptive scheduling of messages on controller area network for real-time control applications. Proc. $1^{\text {st }}$ IEEE Real-Time Technol. Appl. Symp., Chicago, USA, pp. 240-249. 